\title{
PARALLEL RUNNING OF ALTERNATORS.
}

BY ERNEST J. BERG.

In the following article an endeavor will be made to show the principles govering parallel operation of alternators, driven by steam engines or turbines. The laws of parallel running when generators are driven by electric motors depend so much upon the action of the motors that this part of the subject is omitted and reserved for another paper.

Consider two alternators intended for parallel operation, and let:

$e=$ induced $\mathrm{E}$. M. F.

$$
Z_{1}=r_{1}-j x_{1}
$$

$Z_{2}=r_{2}-j x_{2}=$ the internal impedance of the different armatures.

$Y_{1}$ and $Y_{2}=$ the corresponding admittances.

$Z=r-j x=$ the impedance of the external load.

$Y_{3}=$ joint admittance of the armature circuits.

$Z_{3}=$ corresponding impedance.

$Z_{0}=$ total impedance in the circuit.

We have then :

$$
\left.\begin{array}{l}
Y_{1}=\frac{r_{1}}{Z_{1}^{2}}+j \frac{x_{1}}{Z_{1}^{2}} \\
Y_{2}=\frac{Y_{2}}{Z_{2}^{2}}+j \frac{x_{2}}{Z_{2}^{2}}
\end{array}\right\} Y_{3}=Y_{1}+Y_{2}
$$




$$
\begin{gathered}
Z_{3}=\frac{1}{Y_{3}} \\
Z_{0}=Z_{3}+Z
\end{gathered}
$$

Total current $I=\frac{e}{Z_{3}+Z}$

Voltage across load $=I Z=\frac{e Z}{Z_{3}+Z}$

Drop in armatures $=e-\frac{e Z}{Z_{3}+Z}$

Current in armature No. $1=\left(e-\frac{e Z}{Z_{3}+Z}\right) Y_{1}$

Current in armature No. $2=\left(e-\frac{e Z}{Z_{3}+Z}\right) Y_{2}$

Volt-amperes of one armature $=e^{2} Y_{1}\left(1-\frac{Z}{Z_{3}+Z}\right)$

Volt-amperes of one armature $=e^{2} Y_{2}\left(1-\frac{Z}{Z_{i}+Z}\right)$

Power of each armature is the real part of the expression for volt-amperes.

This is expressed as:

and

$$
P_{1}=e^{2} \frac{\left(p r_{1}-q x_{1}\right)}{\left(m^{2}+n^{2}\right)\left(r_{1}^{2}+x_{1}^{2}\right)}
$$

where:

$$
P_{2}=e^{2} \frac{\left(p r_{2}-q x_{2}\right)}{\left(m^{2}+n^{2}\right)\left(r_{2}^{2}+x_{2}^{2}\right.}
$$

$$
\begin{aligned}
p & =m^{2}+n^{2}-m p-n x \\
q & =m x-n r \\
m & =\frac{G}{G^{2}+B^{2}}+r \\
n & =\frac{B}{G^{2}+B^{2}}+x
\end{aligned}
$$




$$
\begin{aligned}
G & =g_{1}+g_{2} \\
B & =b_{1}+b_{2} \\
I_{1} & =g_{1}+j b_{1} \\
Y_{2} & =g_{2}+j b_{2}
\end{aligned}
$$

Neglecting the resistances of the armatures as compared with the reactances, we get the following expressions:

$$
\begin{aligned}
& P_{1}=\frac{e^{2} x_{2} r\left(x_{1}+x_{2}\right)}{Z^{2}\left(x_{1}+x_{2}\right)^{2}+x_{1}^{2} x_{2}^{2}+2 x x_{1} x_{2}\left(x_{1}+x_{2}\right)} \\
& P_{2}=\frac{e^{2} x_{1} r\left(x_{1}+x_{2}\right)}{Z^{2}\left(x_{1}+x_{2}\right)^{2}+x_{1}^{2} x_{2}^{2}+2 x x_{1} x_{2}\left(x_{1}+x_{2}\right)}
\end{aligned}
$$

In other words, the alternators divide the load inversely as their armature reaction.

With a non-inductive external circuit, these questions become:

$$
\begin{aligned}
& P_{1}=\text { power of one armature }=\frac{e^{2} r x_{2}\left(x_{1}+x_{2}\right)}{r^{2}\left(x_{1}+x_{2}\right)^{2}+x_{1}^{2} x_{2}^{2}} \\
& P_{2}=\text { power of other armature }=\frac{e^{2} r x_{1}\left(x_{1}+x_{2}\right)}{r^{2}\left(x_{1}+x_{2}\right)^{2}+x_{1}^{2} x_{2}^{2}}
\end{aligned}
$$

which with close approximation can be written:

$$
\begin{aligned}
& P_{1}=\frac{e^{2} x_{2}}{r\left(x_{1}+x_{2}\right)} \\
& P_{2}=\frac{e^{2} x_{1}}{r\left(x_{1}+x_{2}\right)}
\end{aligned}
$$

Therefore two machines of same induced E. M. F. and armature reaction running in phase will share the load evenly. If one has twice as high armature reaction as the other, it will take one half of the load, etc. Thus since machines of identical regulation have practically armature reactions inversely proportional to their size, it is possible to run such machines from the same engines at proper share of the total load. Should, however, two machines of the same size and different armature reaction run from the same engine, the distribution of power will not be in accordance with the rating. 
To balance the load it is then necessary to add reactance in one armature or to mechanically offset one armature in regard to the other, or, finally, to run them at such field excitation as will cause considerable cross-current, which effect will be discussed later.

In the discussion given above it was assumed that the machines were running strictly in phase, which would practically limit the case to alternators clutched together. If, however, they are rigidly connected together, but out of phase, the current which is due to the resultant of the two E. M. F.'s has a different phase displacement from the E. M. F. of one machine than it has from that of the other, that is, the energy corresponding is different in the two machines, or the one supplies more power than the other.

The relation of currents to E. M. F.'s can also be changed by changing the excitation.

Assume màchines of the same armature reaction which, before being thrown in parallel, have unequal E. м. F.'s. After being connected together their terminal voltages must be the same, therefore a cross-current must flow between them which, in the higher excited alternator, is lagging, and in the other leading; which currents, in conjunction with the field excitation, give a resultant field of same magnitude in the two, but displaced in phase. In the higher excited machine it will bring the resultant field more in phase with the energy current, thus giving a result equivalent to an armature of lower reactance; in the lower excited machine a result same as higher armature reaction. It is therefore possible to change the load by change of field excitation by sacrifice of current, since this method involves wattless currents between the generators.

In view of the above discussion, it is thus evident that the question of distribution of power between alternators is quite simple, if they are running at absolutely the same speed at all loads; that is, if the engines regulate so closely as to permit the clutch to be opened and the armatures still retain the same positions. In reality, however, engines cannot be made to govern so closely, but one will tend to hold up the speed somewhat better than the other. That being the ease, it is safe to say that as a rule the engines will not tend to give the same power at exactly the same speed.

Referring back to the equations and discussions we find, there- 
fore, that with the same armature reaction and induced E. M. F., if different power is given each machine, the armatures must be offset a certain and fixed degree, which means that by even the most minute difference in speed regulation the armatures are forced to change their relative positions, which obviously is a cause for " hunting."

The relative power given by each machine depends upon the respective powers of the engines at identical speeds.

From the above discussion it is apparent that a definite tendency for "hunting" exists, and that this hunting is manifest in a mechanical pulsation in the speed of the armatures, and a transfer of power from one unit to another.

A most superficial investigation will show that this flow of power is much greater than that corresponding to the stored energy of the armature fly-wheel, etc., at the relatively small differences in speed, and that pulsating power actually has to be supplied by the engines. Therefore, and since power cannot be obtained from an engine without corresponding steam, the flow of steam must be periodic to sustain an accumulated hunting.

The remedy is therefore to be found by preventing such periodic flow of steam, which can be accomplished by proper dash pot arrangements at the governor. Other remedies also suggest themselves, the favorite being to make a short-circuited winding in and around the field of alternators. The object of such winding obviously is to establish a load when these oscillations occur, which load tends to stop the acceleration and retardation of the armature. The disadvantages of this method are that losses of no small magnitude not only are introduced when the machines hunt, but also when they are running at steady speed, which losses are caused by induced currents in this short-circuited winding, due to pulsations of flux incidental to modern construction of iron-clad armatures.

Schenectady, October 14th, 1901. 